\title{
Eine Welt oder keine! Plädoyer für ein Weltbürgerrecht als politischer Handlungsauftrag
}

\section{Zusammenfassung}

Die aktuelle Situation des Planeten im Anthropozän verdeutlicht mit Nachdruck: Ein Überleben geht nur gemeinsam! Diese lange bekannte Erkenntnis wird auf der Grundlage eines zukunftsorientierten Weltbürgertums mit historischen Bezügen entfaltet. Das darin enthaltende Innovationspotenzial wird in der Forderung nach einem aufgeklärten Weltbürgerrecht zugespitzt. Dieses orientiert sich an den unveräußerlichen Menschenrechten und einer ökologischen Zukunftsfähigkeit.

Schlüsselworte: Anthropozän, Zukunft, Weltbürgertum, Weltbürgerrecht, Ökologie, Menschenrechte

\begin{abstract}
The current situation of the planet in the Anthropocene shows clearly: A survival of all is only possible as collective approach! This long-known fact is unfolded on the basis of a future-oriented world citizenship with historical references. The innovative potential contained therein is sharpened in the demand for an enlightened world citizenship law. This is based on the inalienable human rights and an ecological viability.
\end{abstract}

Keywords: Anthropocene, future, cosmopolitanism, law of worldcitizenship, ecology, human rights

\section{Anthropozän als Herausforderung}

,Eine Welt oder keine - diese Parole wurde bei den Demonstrationen der Jahre nach 1968 des Öfteren gerufen; auch ich selber habe sie gewiss etliche Male skandiert. Aus welchen politischen Überlegungen dieser Satz abgeleitet war, kann ich heute, rund ein halbes Jahrhundert später, nicht mehr mit Sicherheit sagen - auch sind mir (leider) meine eigenen Gedanken und Assoziationen von damals nicht erinnerlich.

Eines scheint aber heute überaus deutlich zu werden: Im Guten wie im Schlechten ist es diese eine Welt, die wir Menschen gemeinsam reformieren können oder aber ruinieren werden, wobei die Gefahr, dass es dann keine von Menschen bewohnte Erde mehr geben wird, nicht von der Hand zu weisen ist!

Denn für Menschheit und Erde hat am Ende des 20. Jahrhunderts das Anthropozän als neues Zeitalter begonnen, das vor rund 20.000 Jahren begann und auf das postglaziale (nacheiszeitliche) folgte. Das Anthropozän bezeichnet eine Ära, in der die überwiegend nachteiligen Umweltveränderungen der von den Menschen durch die von ihnen selbst geschaffenen Industriezivilisation als Überlebensprobleme der gesamten Menschheit sichtbar werden (Crutzen \& Störmer, 2000).

Das Anthropozän geht, wie mit jedem Tag offensichtlicher wird, mit einer schweren ökologischen Krise einher. Die katastrophalen Folgen dieser Krise treffen die Menschen in den armen Regionen dieser Erde besonders hart. Die reichen Industrienationen hingegen sind weit weniger schwer beeinträchtigt und können sich zudem sehr viel besser gegen die Konsequenzen dieser Krise - wie zum Beispiel gegen die Auswirkungen des Klimawandels - schützen. Eben deshalb scheint es dringlich geboten, auch die Frage nach der Bedeutung der uns vielleicht etwas zu selbstverständlich gewordenen Menschenrechte angesichts der globalen Umweltkatastrophe neu zu stellen. Just dies ist das Thema des vorliegenden Aufsatzes.

\section{Weltbürgertum und Menschenrechte}

Blenden wir deshalb zunächst über 70 Jahre zurück, genau zwei Jahrzehnte vor dem eingangs zitierten Jahr 1968. Im Spätsommer und im Herbst 1948 ging es in Paris noch lebhafter zu als dort ohnehin üblich: Seit dem 11. September tagte in der französischen Metropole die Vollversammlung der Vereinten Nationen, jener neuen Weltföderation, die drei Jahre zuvor, im Juni 1945, von 51 souveränen Staaten gegründet worden war. Der Tagungsort war für „extraterritorial“ erklärt worden, unterlag also nicht mehr der französischen Gerichtsbarkeit. Just dies nutzte ein junger Amerikaner, der damals 26-jährige Garry Davis (1921-2013), der im Krieg Pilot der US Air Force gewesen war. Er hatte am 25. Mai im Pariser Konsulat der Vereinigten Staaten seine US-Staatsbürgerschaft zurückgegeben und sich 
zum ,Weltbürger Nr. 1' erklärt. Um der Ausweisung als staatenloser Ausländer zu entgehen, suchte er Zuflucht auf dem Gelände des Palais de Chaillot, wo die Vereinten Nationen tagten. Er verschaffte sich auch Zutritt zum Tagungsort, wo er am 19. November 1948 nach der Rede des jugoslawischen Delegierten ans Rednerpult trat, das Wort ergriff und sagte: „Herr Vorsitzender, ich unterbreche hier im Namen des Weltvolkes, das hier nicht vertreten ist [...]" Daraufhin wurde er aus dem Saal entfernt. Seine Idee aber gewann alsbald große Popularität - binnen eines Jahres hatten sich 223.801 Männer und Frauen aus 73 Ländern der von Davis initiierten Weltbürgerbewegung angeschlossen; ihr populärstes Gesicht war in der soeben gegründeten Bundesrepublik der Schauspieler Viktor de Kowa (1904-1973).

Garry Davis war gewiss nur einer von vielen Protagonisten einer nach den Schrecken des Zweiten Weltkrieges neu entstehenden Weltbürgerbewegung, wie es sie vor 1914 ja durchaus schon gegeben hatte. Wegen seiner öffentlichen Auftritte im Jahr 1948 ist es aber sicher angemessen, hier und heute seiner besonders zu gedenken.

Die Vereinten Nationen beschäftigten sich damals, im Herbst 1948, mit einer allgemeinen Menschenrechtserklärung - fast 150 Jahre nach der ,Deklaration der Menschenrechte durch die verfassungsgebende Versammlung des revolutionären Frankreichs am 26. August 1789. „Die Menschen werden frei und gleich an Rechten geboren und bleiben es auch", hatte es in jener Deklaration geheißen.

Am 10. Dezember 1948 verabschiedete die UN-Vollversammlung eine neue „Allgemeine Menschenrechtserklärung“, die internationale Gültigkeit für sich beanspruchte. Seit damals gilt der 10. Dezember eines jeden Jahres weltweit als „Tag der Menschenrechte“.

\section{Rechte haben - jenseits nationaler Grenzen}

Im Dezember 1949 veröffentlichte die von den Nationalsozialisten aus Deutschland vertriebene Philosophin Hannah Arendt (1906-1975) in der Zeitschrift ,Die Wandlung' einen Artikel, in dem sie die Meinung vertrat, dass es im Grunde nur ein einziges Menschenrecht gebe, aus dem sich alle anderen Rechte ableiten lassen: das Recht, Rechte zu haben. Im Originalton: „Daß es so etwas gibt wie ein Recht, Rechte zu haben (und das heißt, in einem Beziehungssystem zu leben, wo man nach seinen Handlungen und Meinungen beurteilt wird), oder ein Recht, einer politisch organisierten Gemeinschaft zuzugehören - das wissen wir erst, seitdem Millionen von Menschen auftauchten, die solche Rechte verloren hatten und sie zufolge der neuen globalen politischen Situation nicht wiedergewinnen" (Arendt, 1949, S. 760). ${ }^{1}$

Heute ist es vor allem die globale politische Situation der weltweiten ökologischen Katastrophe, die Millionen von Menschen solche Rechte raubt.

Jenes von Hannah Arendt benannte fundamentale „Recht, Rechte zu haben“ (ebd.) ist auf dem Problemfeld der ökologischen Menschheitsbedrohung gegenwärtig weitgehend inexistent. Dies lässt sich zum Beispiel an einer - an sich gewiss begrüßenswerten - Entscheidung der UN-Vollversammlung vom 28. Juli 2010 zeigen: An jenem Tag hatte das Plenum der Vereinten Nationen auf Antrag Boliviens einmütig (das heißt: ohne eine Gegenstimme, aber bei 41 Enthaltungen) entschieden, das Recht aufZugang zu sauberem Trinkwasser in den seit 1948 verbrieften Katalog der Menschenrechte neu aufzunehmen. Diese Entscheidung weist in einer Zeit, in der, grob geschätzt, rund 900 Millionen Menschen über einen solchen Zugang eben nicht verfügen (und die Zahl der derart Benachteiligten wächst jeden Tag weiter an!), ohne Zweifel in die richtige Richtung; sie bleibt aber zunächst ohne unmittelbare Konsequenzen, da der am Zugang zu sauberen Wasser gehinderte Mensch bislang eben kein Völkerrechtssubjekt ist. Aus seinem abstrakten Menschenrecht auf sauberes Wasser kann deshalb kein konkreter und d.h. alltagspraktisch wirksamer Rechtsanspruch abgeleitet werden, auch wirklich mit sauberem Wasser versorgt zu werden.

Das Grundprinzip eines diesen eklatanten Mangel beseitigenden Weltbürgerrechtes wäre also, abermals mit Hannah Arendt gesprochen, das Recht, Rechte zu haben; zum Beispiel jenes Recht auf Zugang zu sauberem Trinkwasser - und zwar auch (oder gerade) angesichts der ökologischen Bedrohung.

In jener Zeit, in der Hannah Arendt als junge Studentin an verschiedenen deutschen Universitäten jene Anregungen sammelte, aus denen sie später für ihre philosophischen Arbeiten, zum Beispiel zum Thema Menschenrechte, schöpfen konnte, war ein Dokument sehr populär, das in unserer schnelllebigen Gegenwart wohl nur noch wenige Mitmenschen kennen: Der ,Nansen-Pass', der im Jahr 1922 auf Anregung des berühmten Polarforschers und damaligen (ab 1921) Hochkommissars für Flüchtlingsfragen, Fridtjof Nansen (18611930, Friedensnobelpreisträger 1922), vom Völkerbund geschaffen wurde. Dieser Pass sollte den Millionen ,staatenloser ${ }^{6}$ Flüchtlinge wieder zum Recht verhelfen, Rechte zu haben. Im konkreten Fall ging es um das Recht auf Freizügigkeit. Der ,Nansen-Pass` wurde auf Antrag von den Behörden jenes Staates ausgestellt, in dem ein Flüchtling sich aufhielt, hatte ein Jahr Gültigkeit (Verlängerung war möglich) und gab seinem Inhaber das Recht zu reisen und in jenes Land, in dem sein Pass ausgestellt worden war, auch wieder zurückzukehren. Zunächst war der der Nansen-Pass vor allem für Flüchtlinge aus der jungen Sowjetunion gedacht. Er wurde in der Folge auch für armenische (1924), türkische und assyrische Flüchtlinge (1928) ausgestellt sowie ab 1935 auch für Emigranten aus dem Saarland. Prominente Reisende mit einem Nansen-Pass waren Rudolf Nurejew, Igor Strawinski, Marc Chagall und Aristoteles Onassis.

Fast hundert Jahre später, im Sommer 2018, haben Wissenschaftler aus dem Wissenschaftlichen Beirat der Bundesregierung zu Globalen Umweltveränderungen (WBGU) in ihrem Paper Nr. 9 unter der Überschrift ,In Nansens Fußstapfen' einen - so wörtlich - „Klimapass für menschenwürdige Migration“" (WBGU, 2018, S. 24f.) gefordert.

„Migration in Zeiten des Klimawandels ist von der $\mathrm{Na}$ tur der Sache her eine außerordentlich komplexe multikausale und kontroverse Herausforderung “ (ebd.), schreiben die Autoren, und stellen besorgt fest: „Leider ist festzustellen, dass klimabedingte Migration in den letzten Jahren in der internationalen Gemeinschaft zwar zunehmende Aufmerksamkeit erhalten hat, aber die rechtlichen und politischen Lösungsansätze unzureichend sind, um der steigenden Zahl betroffener Personen angemessene, rechtzeitige und gerechte Hilfe zu ge- 
währen“ (ebd., S. 24). Der ,individuelle Ansatz“ (ebd.), wie die Autoren ihn mit dem Hebel des Migrationspasses bewerkstelligen wollen, „soll den einzelnen Menschen in die Lage versetzen, frei über seine Fortwanderung zu entscheiden und stellt insofern den humanistischen Gegenentwurf zur Planmigration dar. Er gibt dem Individuum Würde durch Mobilitätsrecht. Dies ist auch der Ansatz, den der WBGU zur Bewältigung der einsetzenden Klimamigrationskrise empfiehlt. Der WBGU betont, dass sichere und legale Aus- bzw. Einwanderung nicht nur die ultima ratio der Anpassung an den Klimawandel ist, sondern den Betroffenen als Kompensation für die mit ihrem Heimatverlust einhergehenden vitalen und kulturellen Verluste und Schäden auch zusteht" (ebd., S. 25).

Man beachte die Formulierung: auch zusteht - denn damit sind wir wieder beim ,Recht, Rechte zu haben'. Dazu wird gleich unter den Bedingungen der globalen Umweltkatastrophe und der von ihr bewirkten weltweiten Migrationskrise noch Einiges zu sagen sein.

\section{Kants Weltbürgerrecht und die Folgen}

Zunächst lohnt ein Blick auf Immanuel Kant (1724-1804), den ,geistigen Vater' des Weltbürgertums. Er gilt weltweit als der größte Philosoph deutscher Sprache. Sein Traktat ,Zum ewigen Frieden` erschien erstmals im Jahre 1795. Der Königsberger Denker - bei der Veröffentlichung dieser Schrift schon über siebzig Jahre alt - hat in diesem Essay bekanntlich drei ,Definitivartikel` zur Gewährleistung eines ewigen Friedens formuliert. Im ersten dieser Artikel wird gefordert, dass die Verfassung aller Staaten republikanisch sein, d. h. auf der Gewaltenteilung im Sinne Montesquieus fußen solle; im zweiten, dass sich die Staaten der Erde zu einer Föderation, einem ,Völkerbund, zusammenschließen mögen (eine einheitliche Weltrepublik hielt Kant für eher nachteilig und der Despotie förderlich). Zweihundertdreiunddreißig Jahre später kann gesagt werden, dass die Welt diesen ,Definitivartikeln' durchaus nähergekommen ist. Von den souveränen Staaten, die Mitglied der Vereinten Nationen sind, ist gewiss eine sehr viel größere Zahl zumindest dem Buchstaben nach republikanisch verfasst als zu Kants Zeit, als dies ja im strengen Sinne nur von den Vereinigten Staaten von Amerika und von Frankreich nach dem Sturz der jakobinischen Terrorherrschaft hatte gesagt werden können. Und jene Staatenföderation, die Kant seinerzeit angeregt hatte, ist gemäß den berühmten ,14 Punkten' des US-Präsidenten Woodrow Wilson im ,Völkerbund' des Jahres 1919 geschaffen und nach dem recht unrühmlichen Scheitern dieses Bundes in Gestalt der ,Vereinten Nationen' im Jahre 1945 erneut gestiftet worden.

Der Königsberger hat sich insoweit als weit vorausblickender Realist erwiesen, obschon er seinerzeit noch einräumen musste, dass eine Friedensphilosophie wie die seine wohl allgemein verlacht werde.

Es mag allerdings angeraten sein, Kants dritten Definitivartikel näher zu betrachten, dem gerade in Zeiten der ,Flüchtlingskrise' eine besondere Brisanz innewohnt.

In diesem Artikel hatte Kant ein „Weltbürgerrecht“ gefordert, dessen Kern eine „allgemeine Hospitalität“ sein solle. ER fordert darin ein Recht, „welches allen Menschen zusteht, sich zur Gesellschaft anzubieten, vermöge des Rechts des gemeinschaftlichen Besitzes der Oberfläche der Erde, auf der, als
Kugelfläche, sie sich nicht ins Unendliche zerstreuen können, sondern endlich sich doch neben einander dulden müssen" (Kant, 1795/1977, S. 214).

Kant wusste sehr gut, welche Art von Realpolitik diesem Weltbürgerrecht entgegensteht, denn im Gegensatz zu vielen anderen Philosophen seiner Zeit hatte er einen klaren Begriff von der Brisanz jenes Verhältnisses, das wir heute ,Nord-SüdKonflikt' zu nennen pflegen. Vergleiche man, so meinte er, mit der von ihm geforderten weltweiten Hospitalität „das inhospitale Betragen der gesitteten, vornehmlich handeltreibenden Staaten unseres Weltteils, so geht die Ungerechtigkeit, die sie in dem Besuche fremder Länder und Völker (welches ihnen mit dem Erobern derselben für einerlei gilt) beweisen, bis zum Erschrecken weit. Amerika, die Negerländer, die Gewürzinseln, das Kap etc. waren, bei ihrer Entdeckung, für sie Länder, die keinem angehörten; denn die Einwohner rechneten sie für nichts" (ebd., S. 214f.).

Es sei, zwecks besserer Durchdringung des Problems, noch erwähnt, dass Kant sich unter anderem von zwei Entwicklungstendenzen Unterstützung für sein Programm erhofft hat: Von der Entstehung der Weltwirtschaft mit der friedensstiftenden Kraft des Handels und insbesondere „der Geldmacht“ (ebd., S. 16), desgleichen von der Herstellung der „Weltöffentlichkeit“, d. h. von einem Gemeinsamkeitsempfinden, mit dem es so weit gekommen sei, „daß die Rechtsverletzung an einem Platz der Erde an allen gefühlt wird“ (ebd., S. 216).

Unter dem Eindruck der Massaker des Ersten Weltkrieges betonten rund 130 Jahre später etliche europäische Intellektuelle die Bedeutung eben dieses Gemeinsamkeitsgefühls, so etwa der Schriftsteller Stefan Zweig (1881-1942), der den Kriegsbeginn als Augenzeuge miterlebt hatte:

„Wie nie fühlten Tausende und Hunderttausende Menschen, was sie besser im Frieden hätten fühlen sollen: daß sie zusammengehörten [...]“ (Zweig, 1942/2013, S. 299).

Rund zwei Jahrzehnte später, im Jahr der nationalsozialistischen Machtergreifung und nicht lange vor dem Beginn eines neuen Krieges mahnte der Arzt und Kulturkritiker Sigmund Freud (1856-1939) in der Antwort auf einen Brief des Pazifisten Albert Einstein (1879-1955), den dieser im Auftrag des Völkerbundes an ihn geschrieben hatte:

„Alles, was Gefühlsbindungen unter den Menschen herstellt, muß dem Krieg entgegenwirken [...]“ (Freud, 1933/1996, S. 27).

Sehen wir genau hin, erkennen wir allerdings eine durchaus ambivalente Entwicklung. Welthandel und ,Geldmacht' im Sinne Kants haben zwar eine globale Wirtschaftsgemeinschaft erzwungen, innerhalb deren jedoch die schreiendste Ungerechtigkeit herrscht, was auf lange Sicht genügend Anlass für Gewalttätigkeiten aller Art, Unruhen, Revolten und Bürgerkriege bildet - und eben auch für eine immer stärkere Migration. Die Unterhöhlung des Nationalstaates durch diese Art von Weltwirtschaft führt zudem quasi zu einer ,Entstaatlichung' der Kriege, damit auch zur Entwertung der herkömmlichen diplomatischen Methoden zur Schadensbegrenzung, wie dies seither auf vielen Kriegsschauplätzen deutlich sichtbar geworden ist. Dies ist der negative Aspekt jener ambivalenten Entwicklung.

Und auch mit der Weltöffentlichkeit steht es kaum besser. Negativ muss vermerkt werden, dass die gegenwärtige Weltöffentlichkeit zumindest partiell von mächtigen Kartellen 
und Interessengruppen inszeniert wird und dass ihr nur teilweise ein echtes Gemeinschaftsgefühl, häufig aber weit mehr eine Art von sensationsgieriger Schaulust zugrunde liegt, die durchaus dem Diktum aus Goethes „Faust“ zu folgen scheint: „Sie mögen sich die Schädel spalten - Nur in der Heimat bleib's beim Alten!“" (Goethe ,1986 [1808], S. 34).

So bleibt also festzustellen, dass der großartige Kant'sche Entwurf eines Weltbürgerrechtes - als entscheidende Vorbedingung zur Schaffung einer friedlichen Welt - immer noch der Verwirklichung harrt, obschon die globale Lage heute in manchen Einzelheiten günstiger scheint als im Jahre 1795, um einen entscheidenden Schritt weiterzukommen.

\section{Konsequenzen für ein zeitgemäßes Weltbürgerrecht}

Dieser entscheidende Schritt wäre die Schaffung weltbürgerlicher Anrechte, zum Beispiel auf Aus- und Einwanderung, also, mit Hannah Arendt gesprochen, zu der wir damit zurückkehren, das Recht, Rechte zu haben - und zwar auch (oder gerade) angesichts der ökologischen Bedrohung. Noch etwas präziser in Worte gefasst, geht es um den konkreten und damit auch vor Gericht verfolgbaren Rechtsanspruch, sich gegen die Folgen des ökologischen Disasters - etwa die Überflutung des eigenen Wohnortes - zur Wehr setzen zu dürfen. Es ist ja bekannt, dass die Einwohner des pazifischen Inselstaates Kiribati bereits vor geraumer Zeit bei den Vereinten Nationen einen kollektiven Antrag auf Asyl gestellt haben - für den in Bälde zu erwartenden Ernstfall der Überflutung ihres Heimatlandes. Dies ist derzeit nicht mehr als ein reiner Appell ohne rechtliche Bindungswirkung. Genau daran muss sich etwas ändern. Wer, wie die reichen Industrienationen dieser Welt, durch einen luxuriösen Lebensstil leichtfertig die Lebensgrundlagen anderer ruiniert, muss dazu verpflichtet werden, diesen im Ernstfall auch bei der Bewältigung ihrer Notlage behilflich zu sein. Dies wäre im Grunde nichts anderes als eine völkerrechtliche Umsetzung des Verursacher-Prinzips: Polluter pays. Just dies unterstreicht auch der WBGU: „Um die Regelung klimabedingter Migration gerecht zu gestalten, betont der WBGU die zentrale Rolle des Verursacherprinzips. Es sollte die Grundlage für die Entscheidung sein, welche Nationen sich zur Aufnahme von Personen mit Klimapass verpflichten. Länder mit großem Beitrag zum Klimawandel sollten den existenziell Leidtragenden Wege und Rechte für eine würdevolle Zukunft eröffnen. Sie tragen eine erhebliche Verantwortung für die Migrationsursachen der Betroffenen und sollten sich bevorzugt verpflichten, den Inhaber/-innen des Klimapasses Einreiseoptionen zu gewährleisten“ (2018, S. 29).

Wie die noch zu schaffenden Regelungen am Ende en detail auch aussehen mögen - eine Entwicklung in diese Richtung ist jedenfalls nur dann möglich, wenn erstens der ökologisch benachteiligte Mensch zum Rechtssubjekt mit konkreten Rechten geworden ist (eben durch das zu schaffende Weltbürgerrecht) und wenn zweitens Instanzen geschaffen werden, vor denen er rechtliches Gehör finden und sein Recht gegebenenfalls auch durchsetzen kann - nötigenfalls auch gegen Widerstreben. Selbstverständlich müssen dazu unter dem Dach der Vereinten Nationen entsprechende neuartige Institutionen geschaffen werden, aber es gibt keinen einleuchtenden Grund, dessentwegen dies grundsätzlich unmöglich sein sollte, schließ- lich hat die Weltgemeinschaft es ja auch geschafft, den am 17. Juli 1998 gegründeten Internationalen Strafgerichtshof Wirklichkeit werden zu lassen. ${ }^{2}$

Ein ökologisch orientiertes Weltbürgerrecht ist somit ein wesentliches - zugleich freilich ein in seiner konkreten Gestaltung erst noch zu konstruierendes - Werkzeug, wenn die Anpassung an den unaufhaltsamen Klimawandel in einer Art und Weise bewältigt werden soll, durch die weltweit Frieden und Gerechtigkeit gestärkt und gefestigt und nicht beeinträchtigt werden. Als globale Bewältigungsstrategie wäre es wohl mindestens ebenso wichtig, wie der Handel mit Emissionsrechten, die Förderung von erneuerbaren Energien und der Aufbau eines von fossilen Kraftstoffen unabhängigen Verkehrswesens.

Ökologische Rechtssicherheit für alle zu schaffen, ist, mit Kant gesprochen, eine unabweisbare Forderung der Moral, die jedoch, um effektiv wirksam werden zu können, einer umfassenden und dauerhaften Verrechtlichung bedarf: eben in Gestalt jenes Weltbürgerrechts. Dieses Recht muss ein Recht der Einzelperson sein - im Gegensatz zum bisherigen Völkerrecht, im dem alleine die Staaten Rechtsubjekte sind und das Individuum somit im Zustand ohnmächtiger Rechtlosigkeit verharren muss.

Zusammengefasst: Der Imperativ Immanuel Kants: „Es soll kein Krieg sein“, ist ursprünglich ein ethisches Gesetz - ein ethisches Gesetz, das seine eigene dauerhafte Verrechtlichung verlangt. Es bedarf der weltweit gültigen Verankerung dieses Gesetzes in alltagstauglichen Rechtsvorschriften, um durch diese fortdauernd und nachhaltig wirksam werden zu können. „Die konsequente Endgestalt dieser Verrechtlichung ist der eine weltumgreifende Völkerstaat mit Souveränitätsrechten unter den Gesichtspunkten des Friedensgebotes, die äußeren Beziehungen der Einzelstaaten betreffend, und des Republikanismusgebotes, ihre innere Verfassung betreffend" (Dörflinger 2017, S. 319).

Weltbürgerliches Denken bedeutet also, aus Unzufriedenheit mit der gravierenden Ungerechtigkeit unserer Welt und aus einem ethischen Verpflichtungsgefühl heraus eine friedliche und gerechte Welt für alle zu konzipieren. Dazu gehört dann eben auch die global wirksame Verrechtlichung von Menschrechten - wie zum Beispiel der Zugang zu sauberem Trinkwasser - im Sinne eines ökologisch orientierten Weltbürgerrechts. Immerhin stellt die ökologische Krise die größte Bedrohung der Menschen von heute und insbesondere der Armen dar. Sie ist kein in der Zukunft drohender Zustand, sondern ein bereits hier und heute in vollem Gang befindlicher Prozess.

Dieses hier grob umrissene Weltbürgerrecht existiert, wie wir alle wissen, noch nicht. Nur erste Ansätze und Umrisse sind derzeit erkennbar. Das befreit uns freilich nicht von der Verpflichtung, in der eigenen Lebensgestaltung weltbürgerlich zu handeln, wo immer möglich. Und das wiederum heißt, dass wir - wo immer möglich! - vermeiden sollten, auf Kosten anderer zu leben. Denn der glückliche (das heißt: unverdiente) Geburtsvorteil, das Bürgerrecht in einer der reichen Industrienationen zu besitzen, scheint in mehr als einer Hinsicht vergleichbar mit dem glücklich-unverdienten Geburts- und Erbvorteil der Zugehörigkeit zu einem privilegierten Stand, nämlich dem Adel, zu Zeiten der Feudalherrschaft - ein Privi- 
leg, das man sich nicht durch Leistung oder andere Verdienste erworben hat, sondern in das man durch unverdientes Glück hineingeboren worden ist.

Wer also das Glück hat, in eine der reichen Industrienationen - in unserem Fall Deutschland - hineingeboren worden zu sein, sollte daher versuchen, dieses Privileg weltverträglich zu gestalten, was in vielen Fällen ein ,Prinzip Schrumpfung bedeutet, das der sozialschädlichen Ideologie vom angeblich positiven permanenten (Wirtschafts-) Wachstum entgegensteht. So ist es auf Dauer nicht hinnehmbar, dass in einer Industrienation wie Deutschland pro Person und Tag etwa 3.400 kcal Nahrung verzehrt werden - mit einem Anteil von $30 \%$ tierischer Nahrungsmittel, was einem Verbrauch von 3.600 Liter Wasser entspricht. Bei einer vegetarischen (nicht: veganen) Ernährung ließe sich dieser Wasserverbrauch um gut ein Drittel auf etwa 2.300 Liter verringern. Für Weltbürgerinnen und Weltbürger, denen das Schicksal der anderen Menschen auf dieser Erde nicht gleichgültig ist, gilt also klar und eindeutig das ethische Gebot: Weniger Wasser essen (Gerten, 2018).

Was folgt nun aus alledem? In erster Linie wohl dies: Es gibt zwei Grundprinzipien eines weltbürgerlichen Lebens, und das sind diese beiden Leitsätze:

1. Lebe so, dass deine Lebensgestaltung möglichst wenig Schaden für die Lebensführung anderer Menschen und unserer Nachkommen zeitigt!

2. Setze dich dafür ein, dass weltweit eine Rechtsgemeinschaft entsteht, die es auch allen anderen Menschen möglich macht, im Recht zu leben und ihre eigenen Menschenrechte zu verwirklichen.

Handelst du gemäß diesen beiden Grundsätzen, dann - aber nur dann! - darfst du dein Tun und Lassen ein echtes Weltbürgertum nennen!

\section{Anmerkung}

1 Hierzu äußerte sich bereits Kant in seinem Friedenstraktat: „Nur unter der Voraussetzung irgend eines rechtlichen Zustandes (d. i. derjenigen äußeren Bedingung, unter der dem Menschen ein Recht wirklich zu Teil werden kann) kann von einem Völkerrecht die Rede sein..." (Kant, 1795/1977, S. 246).

2 ICC = International Criminal Court; sein Statut trat am 1. Juli 2002 in Kraft, seine Arbeit hat der Gerichtshof am 16. 06. 2003 aufgenommen.

\section{Literatur}

Arendt, H. (1949). Es gibt nur ein einziges Menschenrecht. Die Wandlung, 4, 754-770.

Bastian, T. (2000). Das Jahrhundert des Todes. Zur Psychologie von Gewaltbereitschaft und Massenmord im 20. Jahrhundert. Göttingen: Vandenhoeck \& Ruprecht.

Crutzen, P. J. \& Stoermer, E. F. (2000). Have we entered the „Anthropocene“? Global Change Newsletter, 41, 17-18.

Dörflinger, B. (2017). Rechtliche und ethische Aspekte der Friedensidee bei Kant. In D. Hüning, B. Dörflinger \& G. Kruck (Hrsg.), Das Verbältnis von Recht und Ethik in Kants praktischer Philosophie (Studien und Materialien zur Geschichte der Philosophie 92). Hildesheim: Olms.

Freud, S. (1933/1995). Warum Krieg? Ges. Werke Bd. 16. Frankfurt/M.: S. Fischer. Gerten, D. (2018). Wasser. Knappheit, Klimawandel, Welternährung. München: C.H. Beck. doi.org/10.17104/9783406681349

Goethe, Johann Wolfgang von (1986 [1808): Faust. Der Tragödie erster Teil. In: Werke in 14 Bänden („Hamburger Ausgabe"), Band 3. München: C.H. Beck.

Kant, I. (1785/1977). Grundlegung zur Metaphysik der Sitten. Werkausgabe (hrsg. v. W. Weischedel, Bd. 7). Frankfurt/M.: Suhrkamp.

Kant, I. (1795/1977). Zum ewigen Frieden. Ein philosophischer Entwurf. Werkausgabe (hrsg. v. W. Weischedel, Bd. 11). Frankfurt/M.: Suhrkamp.

Wissenschaftlicher Beirat Globale Umweltveränderungen (WBGU) (2018). Zeit-gerechte Klimapolitik: Vier Initiativen für Fairness. Politik-Papier Nr. 9. Berlin: WBGU.

Zweig, S. (1941/2013). Die Welt von Gestern. Erinnerungen eines Europäers. Köln: Anaconda-Verlag.

\section{Dr. med. Till Bastian,}

Studium der Humanmedizin und praktischer Arzt in Mainz bis 1982; Geschäftsführer 1983-1986 und langjähriges Vorstandsmitglied der IPPNW, Erster Vorsitzender der deutschen Zweiges der Association of World Citizens, freier Schriftsteller, Journalist und Arzt an der psychosomatischen Fachklinik Wollmarshöhe in Bodnegg; Arbeits- und Forschungsschwerpunkte: Psychopathologie, Anthropologie und Friedenspolitik. 